\title{
Thermodynamic analysis of the classical lattice-hole model of liquids
}

\author{
P. Benigni, Aix Marseille Univ., Université de Toulon, CNRS, IM2NP, Marseille, France \\ Institut Matériaux Microélectronique Nanosciences de Provence, UMR 7334 CNRS - Aix-Marseille \\ Université, FST St Jérôme, Service 251, avenue Normandie-Niémen, 13397 Marseille - Cedex 20 (France) \\ Email address: p.benigni@ univ-amu.fr
}

\section{Keywords}

Liquid, glass transition, lattice-hole model, structural order parameter, ortho-terphenyl

\section{Abstract}

A complete thermodynamic analysis of the classical 2-parameter lattice-hole model of liquids is presented. To our knowledge, no such analysis was available before. It is shown that the model depicts a van der Waals like behavior. The calculated phase diagram features a coexistence line between a condensed and a gas phases ending at a critical point. The model is not able to describe a transition between two condensed phases such as melting. Model parametrization to simulate an archetypal fragile glass forming liquid, the ortho-terphenyl, reveals an only qualitative/ semi-quantitative agreement with available experimental information. The extreme simplicity of this 2-parameter model restricts its ability to describe a real liquid. Taking into account the existence of a volume difference between a hole and a molecule, inserting a temperature and pressure dependencies of the physical parameters and allowing the energy parameter to depend on the hole concentration are the most promising modeling options.

\section{Introduction}

Phenomenological approaches relying on the Thermodynamics of Irreversible Processes (TIP) [1] are able to describe the evolution of the configurational part of the thermodynamic functions of vitrifying liquids in the glass transition range. Comprehensive overviews of these approaches can be found in the monographs [2] and [3]. In these approaches, the structural state of the liquid is described by one (or possibly more than one) internal thermodynamic variable(s), also called structural order parameter(s). Under stable or metastable equilibrium conditions, the values of these internal variables are completely defined by the values of the external control variables, temperature and pressure, while, under nonequilibrium conditions, at the glass state, these internal variables become frozen-in.

Within this general framework, a wide spectrum of theories of various generality and complexity were developed by several groups. The requested number of internal variables is classically discussed in relation to the D efay-Prigogine ratio [4], [5], [6], [7], [2], [8], [9]. Without being exhaustive, it is worth mentioning the single structural order parameter approaches of Schmelzer, G utzow, Tropin and coworkers [10], [11], [12], [13], [14], [15], [8] and the one of Garden, Guillou, Richard and Wondraczek [9], [16]. In the following, these two approaches will be referred to by the acronyms SG T and G GRW respectively. More general theories, involving several structural order parameters and the stress/ strain tensor instead of an hydrostatic pressure volume treatment of the mechanical energy, such as the D NLR approach of Cunat and coworkers [17], [18] or the one of Lion et al. [19] were also developed.

The scope of the analysis is now restricted to the theories involving a single structural order parameter. These approaches are build using three main bricks. The first brick is a thermodynamic model allowing to express the $\mathrm{G}$ ibbs energy of the liquid/ glass as a function of the internal, structural order parameter denoted $\xi$, and external, $T$ and $p$, variables. The second brick is a kinetic equation describing the evolution rate of the structural order parameter over time as the liquid gradually departs from equilibrium. From the application of the TIP and entropy production concept, a classical relaxation law is obtained. It expresses that the rate of change of the structural order parameter is proportional to its departure from equilibrium and inversely proportional to a relaxation time. The third brick is a relaxation law giving 
the dependence of the relaxation time as a function of the external and also possibly internal variables.

Let us now focus on the first, thermodynamic model, brick. The first challenge is to define the structural order parameter. If in phase transitions involving crystalline phases, appropriate order parameters can be unambiguously defined e.g. [20], [21], there is no consensus on which structural order parameter should be adopted to best represent the configurational state of a vitrifying liquid/ glass. Hole theory of liquids has a long history since the pioneering works of Eyring [22] and Frenkel [23]. On this basis, several attempts [24], [25], [26], [27], [28], this short list not being exhaustive, were made to describe the glass transition as the freezing process of an equilibrium concentration of holes.

In the SG T and G G RW approaches, the so-called "classical lattice-hole model of liquids" is adopted for the thermodynamic description of the liquid. In this model, the single structural order parameter represents the fraction of free volume. The most detailed presentation of this model can be found in the monograph [3] which is the most useful entry point for anyone interested by this kind of approach. As a complementary tool to reference [3], we propose an extended thermodynamic analysis of the same model. When trying to apply it to a real liquid/ glass, such as in [16] for the extensively studied orthoterphenyl, this in-depth analysis can bring new insight on the physical signification of the model and the choice of the best parametrization methodology.

The structure of the article will be as follows. The main equations and hypotheses of the model are recalled in section II. A complete thermodynamic analysis of the model is provided in section III. The physical interpretation of the model is discussed in section IV. Application of the model to a real liquid, the ortho-terphenyl, and issues linked to the model parametrization are discussed in section $\mathrm{V}$.

\section{Model presentation}

The presentation of the model can already be found in various literature references such as [3] or [8], however, for the sake of completeness, we found relevant to recall in this section the main definitions, assumptions and equations.

The liquid is assimilated to a lattice in which each site is occupied by a particle, which can be, depending on each specific liquid, an atom, a molecule or any structural unit, or a hole. Let us denote $v_{0}$ the volume of a lattice site, considered identical whether or not it is occupied, $N_{0}$ the number of holes per mole of liquid and $N_{A}$ the number of particles per mole of liquid, that is to say the Avogadro number. The volume of an individual site $v_{0}$ can also possibly depends on the external pressure $p$ and temperature $T$ variables and hence become a function $v_{0}(p, T)$.

A structural order parameter, internal variable, $\xi$ representing the fraction of free volume is defined according to:

$$
\xi=\frac{N_{0}}{N_{A}+N_{0}}
$$

This structural order parameter varies between 0 and 1 :

- $\xi=0$ corresponds to the perfectly ordered state, which is the most compact liquid possible without any hole inside.

- $\xi=1$ corresponds to the perfectly disordered state in which all the volume is free, which is the definition of a perfect gas and we will come back latter on this statement.

It is worth noting that, as above defined, the internal variable is rather a disorder parameter than an order one. In the following, we will still call $\xi$ the structural order parameter to follow the generally adopted convention.

The particle fraction $\xi_{A}$ reads:

$$
\xi_{A}=1-\xi=\frac{N_{A}}{N_{A}+N_{0}}
$$


Rewriting the number of holes as a function of the structural order parameter, we also have:

$$
N_{0}=N_{A} \frac{\xi}{1-\xi}
$$

With the former assumptions and definitions, the number of sites per mole of liquid is simply: $N_{A}+N_{0}$ and the corresponding volume:

$$
V(p, T, \xi)=\left(N_{A}+N_{0}\right) v_{0}(p, T)
$$

Which can be rewritten as a function of the structural order parameter:

$$
V(p, T, \xi)=N_{A} v_{0}(p, T) \frac{1}{1-\xi}=V_{0}(p, T) \frac{1}{1-\xi}
$$

Where $V_{0}=N_{A} v_{0}$ is the molar volume of the compact liquid.

If the number of holes is small compared to the number of molecules $N_{0} \ll N_{A}$ and $\xi \approx N_{0} / N_{A}$. This approximation particularly holds at temperatures lower than the melting temperature, hence to a first order, in this temperature range:

$$
V(p, T, \xi) \approx N_{A} v_{0}(p, T)(1+\xi)=V_{0}(1+\xi)
$$

The excess, also called configurational, volume of the liquid containing a certain fraction of holes compared to the most compact liquid possible, having no hole, is by definition: $V^{\operatorname{conf}}(p, T, \xi)=$ $N_{0} v_{0}(p, T)$ and using equation 3 :

$$
V^{\operatorname{conf}}(p, T, \xi)=V_{0}(p, T) \frac{\xi}{1-\xi}
$$

Conversely, from equation 7 , the structural order parameter $\xi$ can be written as a function of the configurational volume:

$$
\xi=\frac{V^{\operatorname{conf}}}{V^{\operatorname{conf}}+V_{0}}
$$

The analytical expression of the thermodynamic functions are now derived starting with the configurational entropy.

Assuming ideal mixing between the holes and the particles, the configurational entropy is written, for a mole of mixture, under the classical form:

$$
S^{\text {conf }}=-R((1-\xi) \ln (1-\xi)+\xi \ln \xi)
$$

$R$ being the gas constant.

The introduction of holes in the liquid modifies the translation, vibration and rotation behavior of the particles. This contribution is not taken into account in the model which only tackles the configurational part of the entropy.

The common practice being to refer the thermodynamic quantities to a mole of matter, hence by dividing the former expression by the number of particles $(1-\xi)$ per mole of mixture, the resulting expression is obtained:

$$
S^{\text {conf }}=-R\left(\ln (1-\xi)+\frac{\xi}{1-\xi} \ln \xi\right)
$$

The number of particles in the system is by definition $N_{A}$. If $z$ is the number of nearest neighbors, also called the coordination number, the probability of finding a hole around a given atom is $z \xi$. D enoting $E_{A A}$ the energy of an AA bond, the configurational internal energy related to the introduction of holes is then written as: 


$$
U^{\text {conf }}=\frac{1}{2} E_{A A} z N_{A} \xi=\Delta E_{0} \xi
$$

Where $\Delta E_{0}=\frac{1}{2} E_{A A} Z N_{A}$.

In this approximation the formation energy of a hole is considered to be independent of the number of holes actually present in the lattice.

The configurational Gibbs energy of the liquid is: $G^{\text {conf }}=U^{\text {conf }}+p V^{\text {conf }}-T S^{\text {conf }}=H^{\text {conf }}-$ TS ${ }^{\text {conf }}$ and combining equations 7, 10 and 11:

$$
G^{\text {conf }}=\Delta E_{0} \xi+p V_{0} \frac{\xi}{1-\xi}+R T\left(\ln (1-\xi)+\frac{\xi}{1-\xi} \ln \xi\right)
$$

The condition of internal equilibrium of the liquid is given by:

$$
\left(\frac{\partial G^{\text {conf }}}{\partial \xi}\right)_{T, p}=0
$$

Inserting expression 12 in condition 13, the equilibrium fraction of free volume $\xi_{e}$ is found:

$$
\ln \xi_{e}=-\frac{\Delta E_{0}\left(1-\xi_{e}\right)^{2}+p V_{0}}{R T}
$$

Or alternatively:

$$
\xi_{e}=\exp \left(-\frac{\Delta E_{0}\left(1-\xi_{e}\right)^{2}+p V_{0}}{R T}\right)
$$

And adopting a dimensionless form:

$$
\xi_{e}=\exp \left(-\frac{\left(1-\xi_{e}\right)^{2}+\chi}{\Theta}\right)
$$

Where:

$$
\begin{aligned}
& \chi=\frac{p V_{0}}{\Delta E_{0}} \\
& \Theta=\frac{R T}{\Delta E_{0}}
\end{aligned}
$$

are dimensionless energies proportional to the pressure and temperature respectively.

By taking the natural logarithm of 16 and rearranging, an alternative and more useful formulation of the internal equilibrium condition is obtained:

$$
\Theta=-\frac{\left(1-\xi_{e}\right)^{2}+\chi}{\ln \xi_{e}}
$$

If, for given values of the external variables $p$ and $T$, equation 19 can only be solved numerically to find the corresponding $\xi_{e}$ value(s), it is seen that if $\xi_{e}$ is rather considered as the independent variable, then, for a given value of $\chi, \Theta$ is an explicit single valued function of $\xi_{e}$ and can be calculated analytically. Or conversely, for a given value of $\Theta, \chi$ is an explicit single valued function of $\xi_{e}$.

The evolution of the equilibrium values of the structural order parameter and the configurational entropy are plotted as a function of $\Theta$ under different isobaric conditions in figures $1 \mathrm{a}$ and $1 \mathrm{~b}$ respectively. 


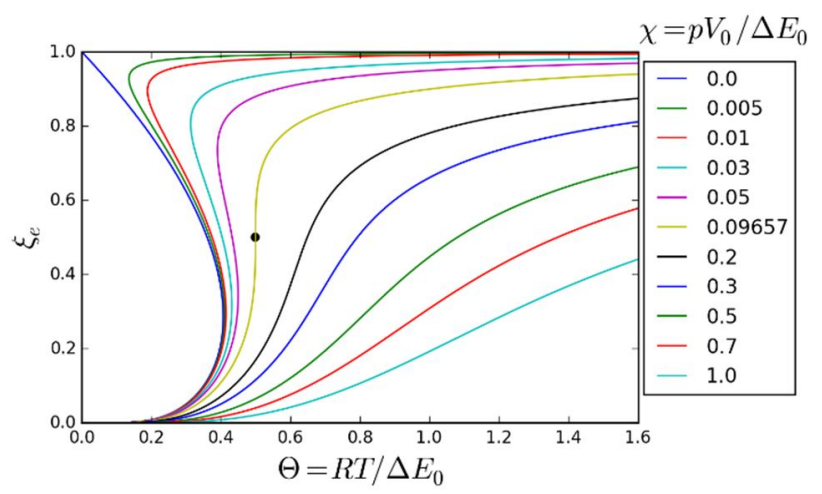

a) Structural arder parameter. Itscitical valueis $\xi_{c}=$ $1 / 2$.

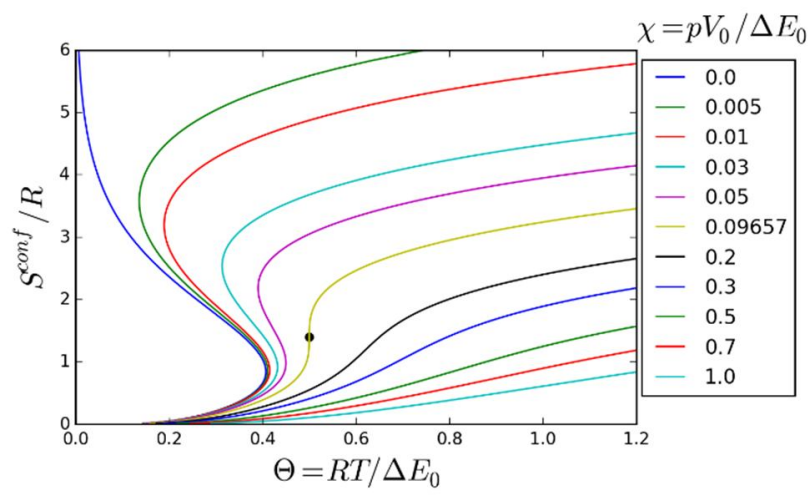

b) Reedured configurational entropy. Its citical valueis $S_{c}^{\text {conf }} / R=2 \ln 2$.

Figre1. Equilibriumvalues of a) thestructural ader paramter andb) theredured configurational etropy vs temperature under isobaric conditions Each arveis labded with its comesponding $\chi$ value The dimensionless citical pressureand temperatureare $\chi_{c}=(2 \ln 2-1) / 4 \approx 0.09657 \ldots$ and $\Theta_{c}=1 / 2$ respetivedy. Thecritical pint is pltted as a badk arde

The shape of the curves evolves as a function of $\chi$, above a critical value of $\chi \approx 0.09657$ the curves are sigmoidal and exhibit a monotonous increase vs. temperature while, below the critical value, they are $\mathrm{S}$ shaped. The representation of figure $1 \mathrm{~b}$ involving the $(T, S)$ pair of thermodynamic conjugate variables is the strict analog, for the thermal energy, of the more familiar $(p, V)$ pair representation for the mechanical energy. Hence, the S shaped $S(T)$ isobars of the model imply a thermodynamic behavior qualitatively similar to the $\mathrm{S}$ shaped $p(V)$ isotherms resulting from the well-known van der Waals equation of state. Another similarity between the present model and the van der Waals equation of state is the number of parameters which is two in both cases.

Before going to the deeper analysis of the model in the next section, let us add that the expressions of all the thermodynamic functions at equilibrium can be derived starting from the general Gibbs energy expression using classical thermodynamic relations combined with the equilibrium condition. The socalled configurational heat capacity at equilibrium $C_{p, e}^{\text {conf }}$ is now derived as an example.

By definition:

$$
C_{p}^{\text {conf }}=\left(\frac{\partial H^{\text {conf }}}{\partial \xi}\right)_{p, T}\left(\frac{\partial \xi}{\partial T}\right)_{p}
$$

The two terms in the right hand side of equation 20 need to be evaluated.

From equation 12, we have:

Then:

$$
H^{\text {conf }}=\Delta E_{0} \xi+p V_{0} \frac{\xi}{1-\xi}
$$

$$
\left(\frac{\partial H^{\text {conf }}}{\partial \xi}\right)_{p, T}=\Delta E_{0}+\frac{p V_{0}}{(1-\xi)^{2}}
$$

The equilibrium condition (equation 14) can be rewritten under the form:

$$
R T=-\frac{\Delta E_{0}\left(1-\xi_{e}\right)^{2}+p V_{0}}{\ln \xi_{e}}
$$

D ifferentiating equation 23 at constant $p$ and after some rearrangements, we obtain: 


$$
\left(\frac{\partial \xi_{e}}{\partial T}\right)_{p}=\frac{R \xi_{e}\left(\ln \xi_{e}\right)^{2}}{\Delta E_{0}\left(1-\xi_{e}\right)\left(1-\xi_{e}+2 \xi_{e} \ln \xi_{e}\right)+p V_{0}}
$$

Then $C_{p, e}^{\text {conf }}$ can be calculated combining equations 20, 22, 24 .

\section{Analysis of the model}

At first, the asymptotic behavior of the internal equilibrium condition represented by equations 14 or 15 is analyzed.

For a highly undercooled liquid, the number of holes becomes negligible $N_{0} \ll N_{A}$ and $\xi_{e} \ll 1$. Equation 15 reduces to:

$$
\xi_{e}=\exp \left(-\frac{\Delta H_{0}}{R T}\right)
$$

Where $\Delta H_{0}=\Delta E_{0}+p V_{0}$.

The classical equation giving the equilibrium concentration of thermal vacancies in a crystal can be found in numerous textbooks such as [29] (page 422) or in more dedicated monograph [30]. It reads:

$$
\xi_{e}=\exp \left(-\Delta G_{0} / R T\right)
$$

Equation 25 is formally equivalent to equation 26 if the entropic contribution to the $G$ ibbs energy of defect formation can be neglected compared to the enthalpic one. This assumption holds true if the number of holes is small compared to the number of particles.

When the fraction of free-volume is close to 1 , starting from equation 14 and using a first-order Taylor expansion of the logarithm:

$$
\lim _{\xi_{e} \rightarrow 1}\left(-\ln \xi_{e}\right)=1-\xi_{e}=\xi_{A, e} \approx \frac{p V_{0}}{R T}
$$

The above equation is the ideal gas law. It is interesting to note that, within the frame of the CALPHAD method [31], Guan and Liu [32] have recently proposed a new physical model to describe thermal vacancies in crystals. Their model is formally very close to the lattice-hole model discussed here. They draw a similar conclusion on the nature of the so-called "vacancy end-member" being an ideal gas.

This simple asymptotic analysis shows that the full range of variation of the structural order parameter $0 \leq \xi \leq 1$ covers the transition from a condensed phase without defect to an ideal gas.

Secondly, the transition between these two physical states can be further analyzed through the determination of the critical point which is defined by the simultaneous nullification of the first three derivatives of the $G$ ibbs energy with respect to the structural order parameter:

$$
\begin{aligned}
& \left(\frac{\partial G^{\text {conf }}}{\partial \xi}\right)_{T_{c}, p_{c}}=0 \\
& \left(\frac{\partial^{2} G^{\text {conf }}}{\partial \xi^{2}}\right)_{T_{c}, p_{c}}=0 \\
& \left(\frac{\partial^{3} G^{\text {conf }}}{\partial \xi^{3}}\right)_{T_{c}, p_{c}}=0
\end{aligned}
$$

Starting from the $\mathrm{G}$ ibbs energy expression (equation 12), the first and second derivatives reads:

$$
\left(\frac{\partial G^{\text {conf }}}{\partial \xi}\right)_{T, p}=\Delta E_{0}+\frac{p V_{0}}{(1-\xi)^{2}}+\frac{R T \ln \xi}{(1-\xi)^{2}}
$$




$$
\left(\frac{\partial^{2} G^{\text {conf }}}{\partial \xi^{2}}\right)_{T, p}=\frac{2 p V_{0}}{(1-\xi)^{3}}+\frac{R T}{\xi(1-\xi)^{2}}+\frac{2 R T \ln \xi}{(1-\xi)^{3}}
$$

From 31 and 28, the following equation holds at the critical point:

$$
\frac{p_{c} V_{0}}{\left(1-\xi_{c}\right)^{2}}+\frac{R T_{c} \ln \xi_{c}}{\left(1-\xi_{c}\right)^{2}}=-\Delta E_{0}
$$

Injecting 33 in 32 and combining with the condition 29, we have:

$$
\left(\frac{\partial^{2} G^{\text {conf }}}{\partial \xi^{2}}\right)_{T_{c}, p_{c}}=-\frac{2 \Delta E_{0}}{\left(1-\xi_{c}\right)}+\frac{R T_{c}}{\xi_{c}\left(1-\xi_{c}\right)^{2}}=0
$$

This expression is pressure independent.

Hence for $\xi_{c} \neq 0$ and $\xi_{c} \neq 1,34$ directly yields:

$$
\xi_{c}\left(1-\xi_{c}\right)=\frac{R T_{c}}{2 \Delta E_{0}}
$$

At the critical point, the third derivative of the free enthalpy with respect to the structural order parameter can be obtained by differentiating 34 with respect to the structural order parameter once. We get:

$$
\left(\frac{\partial^{3} G^{\text {conf }}}{\partial \xi^{3}}\right)_{T_{c}, p_{c}}=-\frac{2 \Delta E_{0}}{\left(1-\xi_{c}\right)^{2}}-\frac{R T_{c}\left(1-3 \xi_{c}\right)}{\xi_{c}^{2}\left(1-\xi_{c}\right)^{3}}=0
$$

Hence again for $\xi_{c} \neq 0$ and $\xi_{c} \neq 1$ :

$$
-2 \Delta E_{0} \xi_{c}^{2}\left(1-\xi_{c}\right)-R T_{c}\left(1-3 \xi_{c}\right)=0
$$

Injecting 35 in 37 , we obtain:

$$
\xi_{c}=\frac{1}{2}
$$

And putting 38 in 35:

$$
\Theta_{c}=\frac{R T_{c}}{\Delta E_{0}}=\frac{1}{2}
$$

As already pointed by Frenkel [23] (page 101), at the critical point, the energy of thermal motion of the particles $R T_{c}$ becomes of the same order of magnitude as the energy required to tear one particle from the rest. With the present model, the heat energy at the critical point is exactly one half of the energy required to create a hole $\Delta E_{0}$.

Note also that the critical values of the structural order parameter and temperature are identical to those of a binary A-B regular solution in which $\xi=X_{A}$ or $X_{B}, X_{i}$ being the molar fraction of $\mathrm{i}$, and $\Delta E_{0}=\Omega$ the regular interaction parameter of the solution, see for instance page 82 of [33].

Combining equations 10 and 38, the critical value of the configurational entropy is:

$$
S_{c}^{\text {conf }}=2 R \ln 2
$$

Substituting 38 and 39 in 33, it is also found that:

$$
\chi_{c}=\frac{p_{c} V_{0}}{\Delta E_{0}}=\frac{2 \ln 2-1}{4} \approx 0.09657
$$

Note that reference [3] only gives a rough estimate $\chi_{c} \approx 0.05$ of the exact critical dimensionless pressure of equation 41. In figures $1 \mathrm{a}$ ) and $1 \mathrm{~b}$ ), the 2 isobaric curves corresponding to the critical pressure have a vertical tangent for the temperature $\Theta_{c}=1 / 2$. 
Finally, substituting 38 in 5 and 7:

$$
\begin{gathered}
V_{c}=2 V_{0} \\
V_{c}^{\text {conf }}=V_{0}
\end{gathered}
$$

According, to Frenkel [23] (page 130), the local range order in a liquid is "tending to zero when the volume becomes about twice as large as $V_{0}$ ", being the volume occupied by one mole of a given substance. Equation 42 is perfectly in line with this estimation and the transition from a solid-like to a gas-like behavior of the liquid at the critical point.

The critical compressibility factor $Z_{c}$ then reads:

$$
Z_{c}=\frac{p_{c} V_{c}}{R T_{c}}=2 \ln 2-1 \approx 0.386
$$

Like for the van der Waals equation of state, $Z_{c}$ has a universal character in the sense that it is found to be independent of the physical parameters of the model. Moreover, its value is close to the $Z_{c}=3 / 8=$ 0.375 value of the van der Waals gas (page 72 of [34]).

Having determined the two pairs of thermodynamic conjugate variables $(-p, V)$ and $(T, S)$ at the critical point, it is more convenient to perform a change of dimensionless variables and to adopt usual definitions of the reduced pressure and temperature.

Let us define:

$$
\begin{aligned}
T^{*} & =\frac{T}{T_{c}} \\
p^{*} & =\frac{p}{p_{c}}
\end{aligned}
$$

Which are simply related to the former dimensionless variables by:

$$
\begin{gathered}
\theta=\frac{1}{2} T^{*} \\
\chi=\frac{2 \ln 2-1}{4} p^{*}
\end{gathered}
$$

Substituting the new dimensionless variables in the internal equilibrium condition 19, we obtain:

$$
\begin{gathered}
T^{*}=-2 \frac{\left(1-\xi_{e}\right)^{2}+\frac{2 \ln 2-1}{4} p^{*}}{\ln \xi_{e}} \\
p^{*}=-4 \frac{\left(1-\xi_{e}\right)^{2}+\frac{\ln \xi_{e}}{2} T^{*}}{2 \ln 2-1}
\end{gathered}
$$

The two above equations allow to explicitly calculate $T^{*}\left(\xi_{e}\right)$ at constant $p^{*}$ and $p^{*}\left(\xi_{e}\right)$ at constant $T^{*}$. The calculated values are plotted by choosing $\xi_{e}$ as the ordinate and, as the abcissa, $T^{*}$ in figure $2 \mathrm{a}$ ) and $p^{*}$ in figure $2 \mathrm{~b}$ ). 


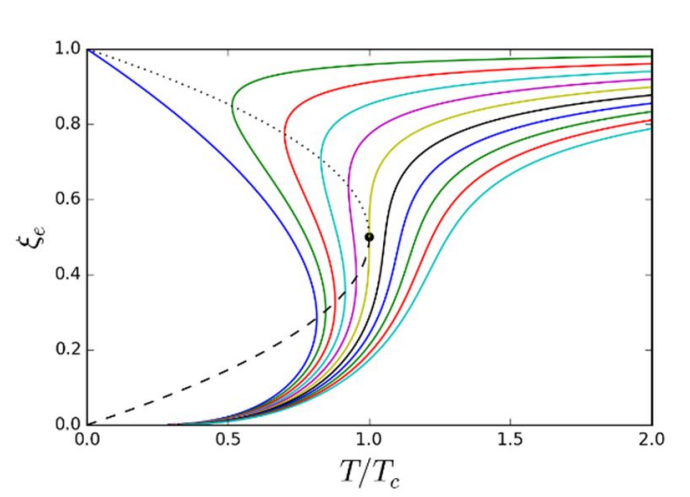

a) Structural ardar paramitervs redured temperature under isdbaric conditions
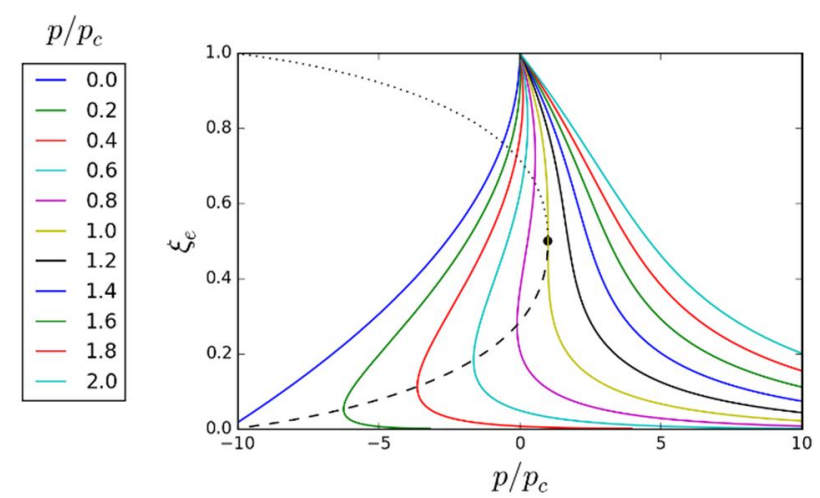

$T / T_{c}$
\begin{tabular}{ll}
- & 0.0 \\
- & 0.2 \\
- & 0.4 \\
- & 0.6 \\
- & 0.8 \\
- & 1.0 \\
- & 1.2 \\
- & 1.4 \\
- & 1.6 \\
- & 1.8 \\
- & 2.0 \\
\hline
\end{tabular}

b) Strutural ader paramter vs reduced pressureunder isthemal conditions

Figre2. Equilibniumvalues of thestructural ardar paramter as a function of a) thereduredtemperatureunder isobaric conditions andb) theredured pressureunder isothemal conditions Thedatted and dashedlines arethetwo branhes of thespinodal arve Theldadk ardeis thecitical paint forwhich $\xi_{c}=1 / 2$.

At equilibrium, the reduced configurational entropy is plotted as a function of the reduced temperature in figure 3a) and the reduced pressure is plotted as a function of the reduced volume in figure $3 \mathrm{~b}$ ).
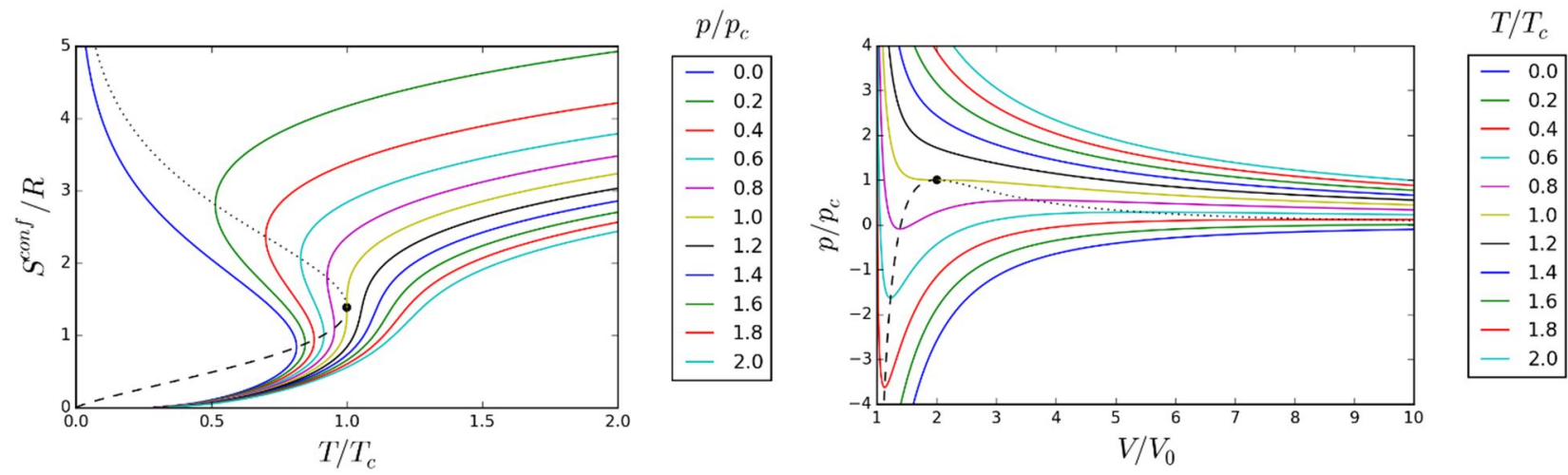

a) Reduced configurational entropyvs redured temperature under isdaricconditions Itscitical valueis $S_{c}^{\text {conf }} / R=$ $2 \ln 2$.

b) Redured pressurevs reduced vdumeunder isthemal conditions Thecitical value of thereduredvdumeis $V_{c} / V_{0}=2$

Figre3. Equilibriumvalues of a) theredured configurational entrqpy as a fundtion of theredreedtemperature under isdbaric conditions andb) thereedred pressureas a funtion of thereduced vdumeunder isthemml conditions The detted and dashedlines arethetwo branches of thespinodal arve Theldad ardeis thecitical paint.

The next step in our analysis consists in determining the spinodal curve. Along this curve, both the internal equilibrium condition 28 and the stability limit condition 29 hold true. These two conditions directly yield equation 35 which hence hold true not only at the critical point but all along the spinodal.

The equation of the spinodal now reads:

$$
\xi(1-\xi)=\frac{R T}{2 \Delta E_{0}}
$$

Substituting 18 and 47 in 51, we obtain the simple expression:

$$
T^{*}=4 \xi(1-\xi)
$$

With 52, the spinodal temperature can be calculated as a single valued function of $\xi$ being the independent variable. Alternatively, if $T^{*}$ is the independent variable, the following quadratic equation must be solved to find $\xi$ values: 


$$
4 \xi^{2}-4 \xi+T^{*}=0
$$

For $T^{*} \leq 1$, the two branches of the spinodal curve are then given by:

$$
\xi_{\text {inf }}=\frac{1-\sqrt{1-T^{*}}}{2} \text { and } \xi_{\text {sup }}=\frac{1+\sqrt{1-T^{*}}}{2}
$$

For each solid curve of figures $3 \mathrm{a}$ ) and $3 \mathrm{~b}$ ), the portion of the curve which is comprised between the two intersections of this curve with the two branches of the spinodal correspond either to:

- $\left(\frac{\partial S}{\partial T}\right)_{p}<0$ negative isobaric heat capacity (figure 3a), which is a condition of thermal instability,

- $\left(\frac{\partial V}{\partial p}\right)_{T}>0$ negative isothermal compressibility (figure $3 \mathrm{~b}$ ), which is a condition of mechanical instability.

In these portions of the curves, representing thermodynamic unstable states, the liquid should break up in a two-phase mixture, these two phases having different values of the structural order parameter, molar entropy and molar volume.

Calculating the stable two-phase equilibria is the final step of our thermodynamic analysis allowing to plot the phase diagram of the lattice-hole model. This is classically done by performing a Maxwell equal area construction on a $p(V)$ isotherm or, equivalently, on a $S(T)$ isobar such as the one shown in 4 . This area matching method is performed numerically by incremental variation of the equilibrium temperature until equal areas are calculated.

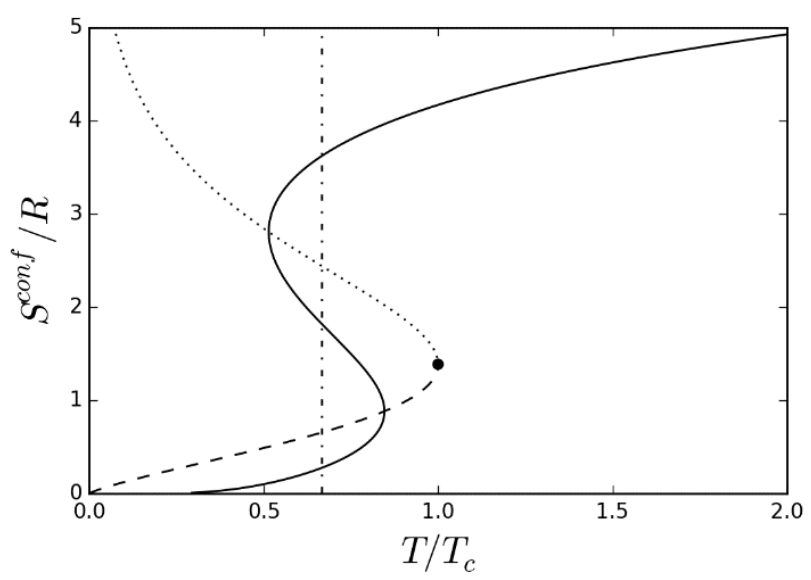

Figre4 Redured configurational entrqpy at equilibriumas a function of theredreed temperaturefor $p / p_{c}=0.2$ (sdid arve). Thedatted and dashed arves arethetwobrandes of thespinodal. Thebladk ardeis thecritical point. The two arees camprised between thevetical dash-dtted lineand thesdidarveareequal. Theredured equilibrium temperatureis $T_{\text {vap }} \approx 0.667$.

As shown by Hillert [35], a 2-D graphical representation is a true phase diagram only if, as axis, one independent variable is taken from each pair in a single set of conjugate pairs. Moreover, a potential diagram, namely a diagram in which all the axis are potential variables ${ }^{1}$ is always a true phase diagram.

The $(p, T)$ phase diagram of the lattice-hole model is presented in figure 5 .

\footnotetext{
${ }^{1}$ For the heat, mechanical and chemical energy forms in the energy scheme, the corresponding potential variables are respectively the pressure (with a minus sign) $-p$, temperature $T$ and chemical potential. The potential variables are intensive. In contrast to the molar variables, such as the molar entropy, molar volume or molar fraction which are also intensive, at equilibrium in a multiphase, multicomponent system, the pressure, temperature and chemical potential have uniform values in all parts of the system.
} 


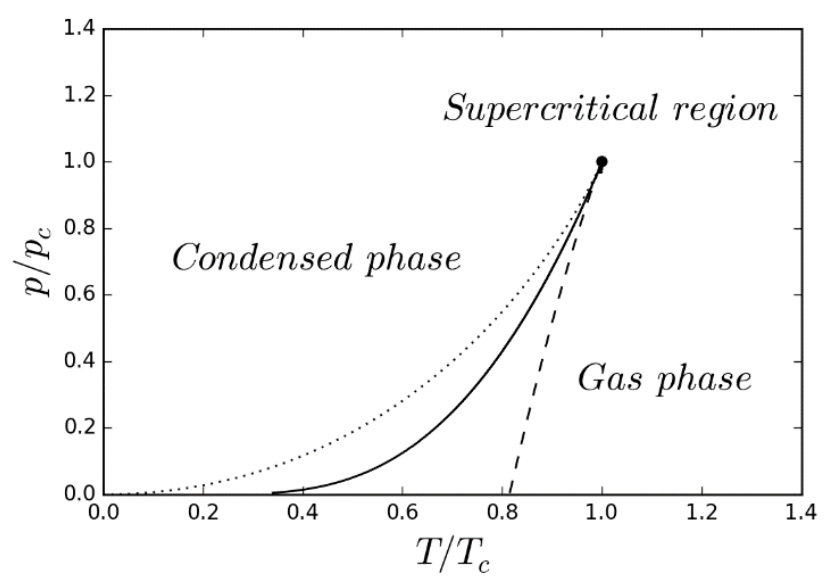

Figure5. $(p, T)$ phasediagramof thelatticehdemodd. Thesdid arverepresentsthetwophaseequilibriumline The two cexistingphases alongthismmovariant linearea condensed oneanda gas likeone Theddted and dashed aurves are thetwo brandhes of thespinodbl. Thethreearvesjoin at thecritical point (bladk irde) with a common tangent. The phasediagamshous the possibility of a supercitical continuaus transition between thecondensed and gaslikephases Theares comprised between the two spinodal brandes and thecoexistencearvedefinetheextensin of themtastability domins of thecondensed and gas phases (seetext bedow).

The area between the dashed and the solid curves is a zone of potential metastable existence of the condensed phase. At the right of the dashed line, the condensed phase is unstable and cannot exist. The area between the dotted and the solid curves is a zone of potential metastable existence of the gas phase. At the left of the dotted line, the gas phase is unstable and cannot exist.

\section{Physical interpretation of the model}

According to the analysis presented in the previous section, the model depicts, below the critical point, a first order transition between a condensed phase and a gas phase and a continuous transition between these two states in the supercritical region. This analysis is consistent with the alternative name sometimes adopted for this model: the lattice-gas model. A prion, the condensed phase can be chosen to be a liquid, as in the works of the SGT and G GRW groups, or a solid, as in the work of [32].

However, the model is not adapted to describe the melting transition which involves two condensed phases. Several arguments that can substantiate this conclusion have been given long ago by Milchev and Gutzow [36] and presented more recently in a concise form by Gutzow and Schmelzer in [3]. These arguments are of both qualitative and quantitative natures. In the first category, they remind that phases such as the crystal and the liquid have qualitative differences in terms of symmetry preventing the possibility of a continuous transition between them. We would like to further elaborate from another more quantitative argument the fact that, at a given temperature, the number of vacancies in a crystal is smaller than the number of holes in the liquid.

First, if the condensed phase is considered to be the liquid, the physical parameters $\Delta E_{0}$ and $V_{0}$ of the model must be adjusted to calculate a fraction of holes at the melting point which is around 5-10\% under atmospheric pressure as can be crudely estimated from the difference of the molar volumes of the crystal and liquid phases at the melting temperature for a large number of substances. In particular, for closepacked hcp and fcc metals, Hillert (page 423 of [29]) points out that the coordinence is 11 in the liquid while it is 12 for the crystal, the difference being $1 / 12 \approx 8 \%$. This estimation is also consistent with Frenkel's statement ([23] page 130) that "a complete disappearance of long distance order in the arrangement of the particles can take place only when the number of atomic holes reaches a certain fraction - a few percent say - of the number of particles". If alternatively, the condensed phase is considered to be the crystal, the physical parameters $\Delta E_{0}$ and $V_{0}$ of the model must rather be adjusted to fit the fraction of thermal vacancies in the crystal. The equilibrium concentrations of vacancies at the melting point have been extensively studied in pure metals [37] and are in the range $0.1-0.01 \%$ for low melting point ones. As an example, for aluminum, the measured concentrations spans from 0.06 up to 
$0.2 \%$ depending on the measuring technique (see table 11.1 of [37]) while the liquid porosity estimated from the difference between the molar volumes of the liquid and the crystal is around 7\% [38] at the melting point. Hence, the concentration of holes in the liquid is in this case at least 30 up to possibly 100 times larger than in the crystal. It is concluded that the parameters of the model can only be adjusted to fit one of these very different concentrations of defects. It results that if the liquid phase of a given substance is to be modeled as a lattice, the properties of this lattice should be different from the ones of the corresponding crystal lattice and that two distinct lattices are then required to model a crystal/ melt transition as proposed in [36].

The difference between the real crystalline lattice and the liquid quasi-lattice which arises from the two phases having different coordination numbers and different interatomic or intermolecular distances should be traduced in terms of the only two $\Delta E_{0}$ and $V_{0}$ parameters of the model. At a given temperature, for most liquids, the molar volume of the liquid is expected to be larger than the one of the crystal and the volume of the compact liquid with no hole $V_{0}$ should also be larger than its crystalline counterpart. The choice of these parameters to model a specific liquid is discussed in the next section.

\section{Model parametrization and application to a specific liquid}

We will start by comparing the parametrization strategies in the SGT and GG RW approaches.

In all the works of the SG T group, a first simplification of the Gibbs energy expression 12 is performed for numerical applications. With the assumption $\xi \ll 1$ which holds true in the glass transition range at temperatures significantly lower than the melting temperature, the enthalpic contribution to the $\mathrm{Gibbs}$ energy can be approximated by:

$$
H^{\text {conf }}=\Delta E_{0} \xi+p V_{0} \frac{\xi}{1-\xi} \approx \Delta E_{0} \xi+p V_{0} \xi=\Delta H_{0} \xi
$$

Where $\Delta H_{0}=\Delta E_{0}+p V_{0}$. Combining 55 with 10 , the G ibbs energy expression now reads:

$$
G^{\text {conf }}=\Delta H_{0} \xi+R T\left(\ln (1-\xi)+\frac{\xi}{1-\xi} \ln \xi\right)
$$

This simplified expression has a single parameter and only one piece of information is required to adjust its value. These authors do not apply the model to a real liquid but rather to a fictive one having typical properties of vitrifying substances. The value of $\Delta H_{0}$ is adjusted in order to obtain $\xi_{e} \approx 0.05$ at the melting temperature chosen to be $750 \mathrm{~K}$ for this hypothetical substance. This $5 \%$ value is consistent with the molar volume difference which is observed for many substances between the crystal and the liquid at the melting point as already discussed in section IV. In doing this, the authors implicitly assimilate the molar volume of the compact liquid $V_{0}$ to the molar volume of the crystal at the melting temperature:

$$
V_{0} \approx V_{c}\left(T_{m}\right)
$$

The validity of this assumption requires to be further checked.

GGRW [16] have applied the model to the ortho-terphenyl (o-TP, $\mathrm{C}_{18} \mathrm{H}_{14}$ ). This substance has been extensively studied in the glass research community because its melting temperature is $329.354 \mathrm{~K}$ [39] hence the temperature range of the super-cooled liquid is around the ambient temperature [40]. Moreover, it can be vitrified under moderate pressures. Its molecule is relatively symmetrical, rigid, compact and non-polar with a shape quite similar to a spherical particle [41]. This liquid is an archetypal fragile one in which the rigid molecular units interact by weak non-directed van der Waals type forces. The three-step parametrization methodology adopted in [16] is now described and critically reviewed.

First, the equilibrium fraction of free volume at the melting point $\xi_{e}\left(T_{m}\right)$ is estimated on the basis of the molar volume difference between the liquid and crystalline 0-TP according to: 


$$
\xi_{e}\left(T_{m}\right)=\frac{V_{l}\left(T_{m}\right)-V_{c}\left(T_{m}\right)}{\left(\frac{V_{l}\left(T_{m}\right)+V_{c}\left(T_{m}\right)}{2}\right)}
$$

Using the molar volumes at the melting point under atmospheric pressure from [42], it is found that $\xi_{e}\left(T_{m}\right) \approx 0.0928$. This estimation again relies on the assumption 57 . However, considering equation 8 , a more consistent estimation of the structural order parameter at $T_{m}$ would be:

$$
\xi_{e}\left(T_{m}\right)=\frac{V_{l}\left(T_{m}\right)-V_{c}\left(T_{m}\right)}{V_{l}\left(T_{m}\right)}
$$

This criticism remains minor since adopting equation 59 only modifies the value at the margin giving $\xi_{e}\left(T_{m}\right) \approx 0.0887$ which is still around $9 \%$.

Second, knowing $\xi_{e}\left(T_{m}\right), V_{0}$ is estimated using the Clausius-Clapeyron relation applied along the crystalliquid equilibrium line to be $V_{0}=7.34 \mathrm{~cm}^{3} \mathrm{~mol}^{-1}$. This value is abnormally low considering that i) the molar volume of the liquid is $V_{l}\left(T_{m}\right)=218.51 \mathrm{~cm}^{3} \mathrm{~mol}^{-1}$ [42] under atmospheric pressure and ii) if $\xi_{e}\left(T_{m}\right) \approx 9 \%$, equation 5 implies that $V_{0} \approx 0.91 V_{l}\left(T_{m}\right)$. This is an objection to the parametrization method retained by G G RW. In fact, as explained in section IV, the model does not describe the crystalliquid equilibrium but the liquid-gas one. Thus, injecting the entropy of melting and the corresponding molar volume change in the Clausius-Clapeyron equation, as done in equation 8 of [16], does not seem to be justified.

Third, injecting the $\xi_{e}\left(T_{m}\right)$ and $V_{0}$ values in the internal equilibrium condition (equation 14 ), $\Delta E_{0}$ is estimated to be $7909 \mathrm{~J} \mathrm{~mol}^{-1}$ by GGRW.

At this stage, it is interesting to look for an alternative method of identifying $V_{0}$ for the o-TP on the basis of dilatometric measurements of the liquid molar volume available in literature [43], [44], [42]. Unfortunately, the data points are not given in the original papers and the results are rather only given as polynomial fitting equations.

According to [43], the liquid molar volume reads:

$$
V=V_{l}\left(T_{m}\right)\left(1+\alpha_{l}\left(T-T_{m}\right)\right)
$$

In which $V_{l}\left(T_{m}\right)=218.6 \mathrm{~cm}^{3} \mathrm{~mol}^{-1}, \alpha_{l}=7.2510^{-4}{ }^{\circ} \mathrm{C}^{-1}$ and $T_{m}=55.5^{\circ} \mathrm{C}$.

The regression equation of [44] is:

$$
V=0.911+6.4610^{-4} T+6.410^{-7} T^{2}
$$

In which $V / \mathrm{cm}^{3} \mathrm{~g}^{-1}$ and $\mathrm{T} /{ }^{\circ} \mathrm{C}$.

The two above experimental studies were performed under atmospheric pressure. Naoki and Koeda [42] have investigated the combined effects of pressure and temperature. Their regression polynomial reads:

$$
V=\sum_{i=0} \sum_{j=0} c_{i, j} T^{i} p^{j}
$$

The elements of the $c_{i, j}$ matrix being given in the original paper.

Equations 60, 61, 62 are graphically compared in figure 6 showing the consistency between the three dilatometric studies under atmospheric pressure. At first order, the volume can be considered to increase linearly with temperature meaning that the magnitude of the quadratic or higher order terms in equations 61 and 62 remains small. 


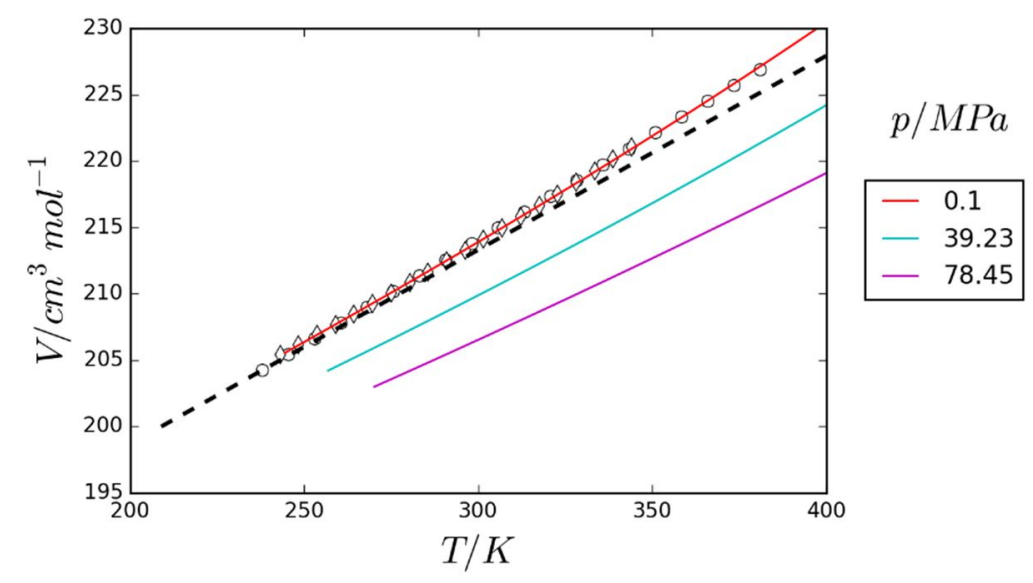

Figure6. Mdar vdume of liquidoTP mesureel by dilatomeryvs temperature Nadki andKoedaresults (equation 62) areplotted as solid lines for the threpressures investicated. Under atmosphericpressure, a good agreement is dbserved with theresults of Andress andUnbbdhode(inde) andGret andTumbull (diamonds). Notethat for thesetwolast studies, thesymbdsare dotted usingreyession equations 60 and 61 and arend theexpeimental pints thenselves not given in theorignal papers Themodd of Bartos andKristiak (equation 63, dashedline) validfor $T<290 \mathrm{~K}$ is extrapdated upto $400 \mathrm{~K}$

Bartos and Kristiak [45] proposed to express the liquid molar volume under a linear form analog to equation 60:

$$
V=V_{i}\left(1+\alpha_{l}\left(T-T_{i}\right)\right)
$$

Where $\alpha_{l}$ is the mean thermal expansion coefficient, $T_{i}$ is the so-called initial temperature at which the free volume begins to appear and $V_{i}$ the liquid molar volume at $T_{i}$. Combining the dilatometry results of [42] with the positron annihilation lifetime spectroscopy results of [46], they were able to determine the values of the three parameters in the above equation.

A mean thermal expansion coefficient $\alpha_{l}=7.3110^{-4} K^{-1}$ is derived by Bartos and Kristiak between 250 and $290 \mathrm{~K}$. Their value is in the range of other literature ones: $\alpha_{l}=7.2510^{-4} \mathrm{~K}^{-1}$ [43] and $\alpha_{l}=$ $7.34410^{-4} \mathrm{~K}^{-1}$ [42]. The two other parameters are $T_{i}=209 \mathrm{~K}$ and $V_{i}=200.0 \mathrm{~cm}^{3} \mathrm{~mol}^{-1}$. It is seen from figure 6 , that equation 63 starts to deviate from the experimental results when extrapolated between 300 and $400 \mathrm{~K}$ because of the non linear character of the volume increase over an extended temperature interval. A better agreement could be achieved in the whole $210-400 \mathrm{~K}$ range, somewhat artificially, by increasing the mean thermal expansion $\alpha_{l}$ up to $7.810^{-4} K^{-1}$ keeping other parameters constant. Bartos and Kristiak point out the good agreement between $T_{i}=209 \mathrm{~K}$ and the Kauzmann temperature of 0 -TP: $T_{K}=200 \pm 10 K$.

The value of $V_{i}$ is of greater practical interest for the present study because, by definition, it identifies with $V_{0}$ the volume of the compact liquid without hole in the lattice-hole model. Comparing $V_{i}=V_{0}=$ $200.0 \mathrm{~cm}^{3} \mathrm{~mol}^{-1}$ and $V_{c}\left(T_{m}\right)=199.13 \mathrm{~cm}^{3} \mathrm{~mol}^{-1}$ from [42] it is concluded that for 0 -TP, at least under atmospheric pressure, equation 57 holds true and provides a very simple and efficient way to estimate $V_{0}$ which additionally guarantees its consistency with the $\xi_{e}\left(T_{m}\right)$ value.

Substituting $V_{0}=199.13 \mathrm{~cm}^{3} \mathrm{~mol}^{-1}$ and $\xi_{e}\left(T_{m}\right) \approx 0.0887$ in the internal equilibrium condition (equation 14), $\Delta E_{0}$ is then estimated to be $7964 \mathrm{Jmol}^{-1}$. This parameter set is compared to the one adopted by GG RW in table 1. D espite the large difference in the $V_{0}$ value between the two studies, the $\Delta E_{0}$ values are quite close because, under atmospheric pressure, $p V_{0} \ll \Delta E_{0}\left(1-\xi_{e}\left(T_{m}\right)\right)^{2}$ regardless of the value of $V_{0}$. Hence, the evolutions of the structural order parameter at equilibrium calculated with the two parameter sets of table 1 in the 100-350 K temperature range are also very similar as can be seen in figure 7. 
Table1. Paramtes of thelatticehdemod for theoTP liquid under $0.1 M P a$.

\begin{tabular}{|c|c|c|c|}
\hline Reference & $\xi_{e}\left(T_{m}\right)$ & $V_{0} / \mathrm{cm}^{3} \mathrm{~mol}^{-1}$ & $\Delta E_{0} / \mathrm{J} \mathrm{mol}^{-1}$ \\
\hline GGRW [16] & 0.0928 & 7.34 & 7909 \\
\hline This work & 0.0887 & 199.13 & 7964 \\
\hline
\end{tabular}

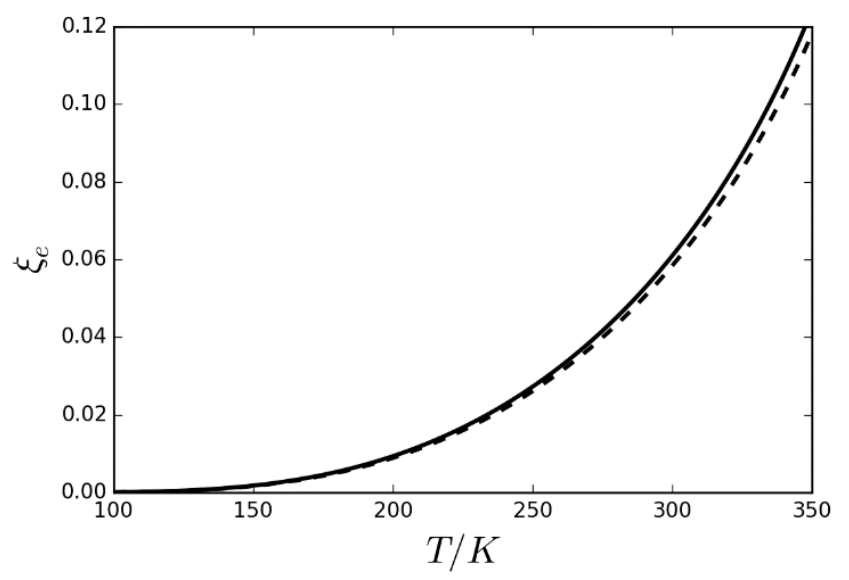

Figure7. Structural arder parameter at equilibriumvs temperatureaccordingtoGGRW (sdidline) and this work (dashedline). Thests of paramters used tocalailatethetwo arvesaregiven in table1.

The validity of our parameter set is now further checked towards various available experimental quantities. We can calculate $\Delta H_{0}=\Delta E_{0}+p V_{0} \approx 7984 \mathrm{Jmol}^{-1}$. When $\xi \ll 1$ holds true, this quantity is the single parameter of the configurational enthalpy (see equation 55) and, as such, can be compared to the vaporization enthalpy of the 0 -TP. According to Verevkin [47], $\Delta_{v a p} H=81 \pm 0.9 \mathrm{kJmol}^{-1}$ at the average temperature $\bar{T}=352 \mathrm{~K}$ near the o-TP melting point. Hence, we have: $\Delta H_{0} \approx 0.1 \Delta_{v a p} H$. This order of magnitude is consistent with the parametrization adopted by the SG T group choosing as a rule of thumb $\Delta H_{0} \approx 0.166 \Delta_{v a p} H$ for the numerical calculations.

Then the critical temperature, pressure and volume can be evaluated using equations 39, 41 and 42 respectively and compared with the IUPAC recommended values for 0-TP [48] in table 2. The critical pressure is quite well estimated by the model while the critical temperature and volume are highly underestimated.

Table2. Critical temperature, pressure vdumeand compressibility factor of liquid/ vapar oTP as calaulated fromthe latticehdemodd with thest of paramtes given in table1 and compared with IUPAC recommended values

\begin{tabular}{|c|c|c|c|c|}
\hline Reference & $T_{c} / K$ & $p_{c} / \mathrm{MPa}$ & $V_{c} / \mathrm{cm}^{3} \mathrm{~mol}^{-1}$ & $Z_{c}=\frac{p_{c} V_{c}}{R T_{c}}$ \\
\hline This work & 479 & 3.86 & 398 & $2 \ln 2-1 \approx 0.386$ \\
\hline IUPAC [48] & $857 \pm 5$ & $2.99 \pm 0.6$ & 731 & 0.307 \\
\hline
\end{tabular}

The molar volume calculated with the model is compared to the measurements of Naoki and Koeda in figure 8. The calculated curve (dashed black line) tends towards $V_{0}$ with a horizontal asymptote for temperatures lower than $150 \mathrm{~K}$. D ue to the adopted parameter set, the calculated (dashed black line) and the measured (blue solid line) curves intersect at the melting temperature (black circle). It is also seen that the calculated volume increases more strongly with temperature than the measured one. This behavior can be understood considering equation 6 . It expresses that the volume is, in a first approximation, a linear function of the structural order parameter. However, the structural order parameter is itself a strongly non-linear function of the temperature as shown in figure 7. As a consequence, the calculated volume is also a non-linear function of temperature whereas the measurements follow a globally linear 
trend consistent with equations 60 or 63.

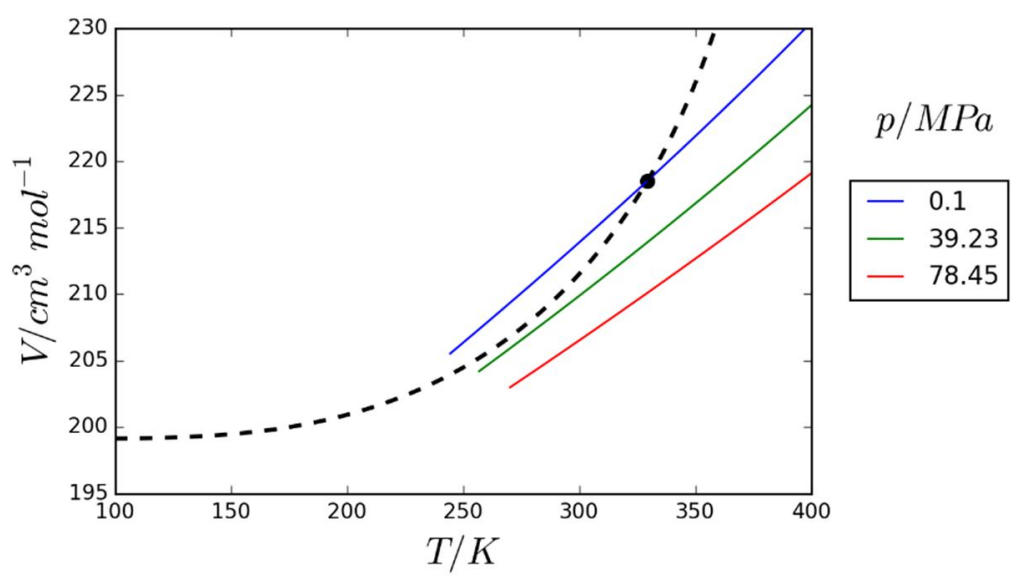

Figre8. Mdar vdumeof theliquidat equilibriumvs tempeature Meesuremets of Nadki and Koeda(sdidlines) compared to thearvecalailated with themodd (dahhed bladk line) under atmospheic pressure using thest of paramtes from this work as givenin table 1. Thedashed bladk and sdid bue anvesinteseet at themdtingpaint (dadk arde).

Attempts were also made to parametrize the model for $p=39.23$ or $78.45 \mathrm{MPa} . V_{C}\left(T_{m}\right)$ and $V_{l}\left(T_{m}\right)$ values under these elevated pressures were taken from the work of Naoki and Koeda [42]. It was further assumed that equation 57 was still valid in this pressure range. However, negative $\Delta E_{0}$ values were calculated which does not seem to be a reasonable result considering that the energy required to form a hole must be endothermic.

Finally, combining equations 10 and 15, the configurational entropy of the supercooled liquid in metastable equilibrium is calculated with the model as a function of temperature under atmospheric pressure and plotted in figure 9. The entropy difference between the liquid/ glass and the crystal derived from the calorimetric study of Chang and Bestul (see table II of reference [39]) is plotted on the same graph for comparison. The slope of the entropy rise just above the glass transition temperature at $240 \mathrm{~K}$ is close to the experimental one but the overall agreement is only qualitative.

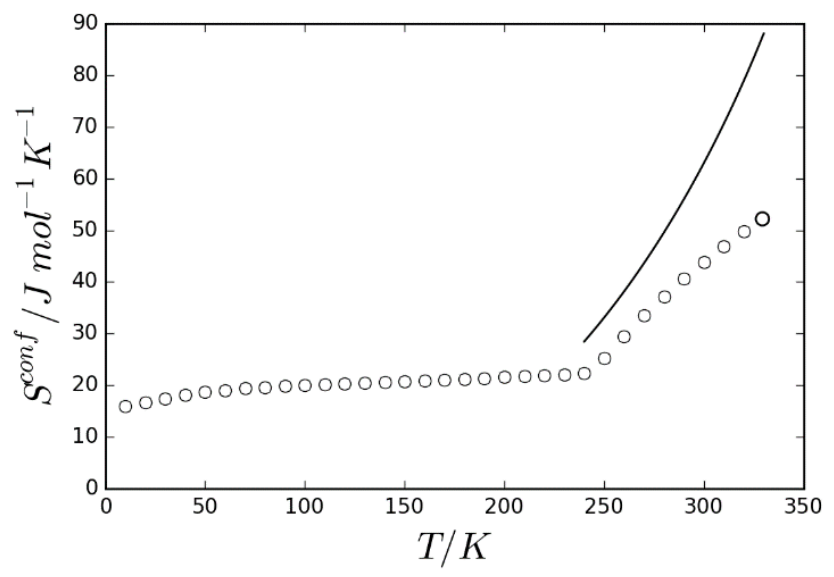

Figre9. Configurational entropy of thesuperooled liquid calailated vs temperaturewith themodd (sdidline) under atmospheicpressure using thest of paramtersfromthis vork as given in table1. Thecalaulation is peformed between thegass transition (240 K) and themdting(329.352 K) temperatures Entropy differencebduentheliquid gass and theorystal deivedfromthecaloimenicsurdy of Changand Bestul (emptydirdes). Themdeof substanceis defined as $\mathrm{C}_{18} \mathrm{H}_{14}$. 


\section{Conclusions and outlook}

A complete thermodynamic analysis of the classical two-parameter lattice-hole model of liquids is provided in the present work. To our knowledge, such analysis was not available before in literature. It has been shown that this model depicts a van der Waals like behavior. The $(p, T)$ phase diagram of the model has been calculated. It features a coexistence line between a condensed and a gas phase which terminates at a critical point.

Attempts were performed to parametrize the model for simulating an archetypal fragile glass forming liquid, the ortho-terphenyl. Under atmospheric pressure, the comparison with available experimental information in term of molar volume and entropy have shown that the agreement is at best of qualitative/ semi-quantitative nature. At higher pressures, no satisfying parametrization of the model could be achieved.

In this work, only the simplest version of the lattice hole model with two physical parameters considered constant was tested. It is very likely that the extreme simplicity of this model restricts its ability to describe a real liquid and that more sophisticated modeling approaches, necessarily having more parameters, are required.

Among some possible refinements, let us mention some important modeling options based on physical considerations.

First, it seems necessary for some liquids to take into account the existence of a volume difference between a hole and a molecule. In particular, for the ortho-terphenyl, Bartos and Kristiak [45] have evaluated the ratio $n=v_{h} / v_{0}$ between the volumes of a hole $v_{h}$ and of a molecule $v_{0}$ to vary between 23.2 and $62.2 \%$ in the $243-304 \mathrm{~K}$ temperature range. In their lattice-hole models of polymeric substances, Hirai and Eyring [24] and Smith [26] have indeed introduced such volume ratios taking $n$ values in the range $1 / 6-1 / 5(17-20 \%)$.

Second, a major improvement could be achieved by considering that the physical parameters are themselves functions of the intensive thermodynamic variables $p$ and $T$. Starting with the volume parameter $V_{0}$, Frenkel mentions [23] (page 175) that "in reality, the free volume of a liquid body must be distributed partly in a discontinuous way in the form of separate holes, and partly in a continuous way in the form of a general increase of the average distances between the particles in those regions which preserves their homogeneity". Coming back to the ortho-terphenyl case, the linearity of the volume increase with temperature shown by figure 6 and consistent with equations 60 or 63 tends to indicate that the thermal expansion results more likely from a uniform increase of the interparticle distances than from the introduction of holes in the liquid structure. This conclusion is fully consistent with the analysis of Bartos and Kristiak [45] considering that the volume of the supercooled ortho-terphenyl is a simple thermal expansion of the initial volume $V_{i}$ at $T_{i}$ and that the main contribution to the growth of the volume fraction of holes is the increase of the mean free volume hole size and not the increase of the number of holes. Trying to rationalize the effects of both the temperature and pressure in a simple way, the volume parameter functional dependence could be, following Smith [26]: $V_{0}=V_{00}(1+a T)(1-$ $b p$ ) with $a, b>0$. Considering now the energy parameter $\Delta E_{0}$, a simple temperature dependence in the form $\Delta E_{0}=a+b T$ could be used in order to model the entropy change other than configurational caused by introducing holes in the liquid structure.

Third, following Milchev and Gutzow [36], it is worth wondering "to what extent the bond energy between two nearest neighbors in a lattice depends on whether neighboring sites are occupied or free". To account for this effect, the energy parameter could also be rendered dependent of the hole concentration. In this respect, analytical forms could be adopted from the CALPHAD thermodynamic modeling of solutions [31].

The above modifications are thought to be the simplest and natural ones that could improve the model ability to quantitatively describe a real liquid. More drastic changes in the modeling approach, such as the introduction of an additional order parameter [49], could also be of interest but are outside the scope of the present article. 


\section{Acknowledgments}

Jacques Rogez (IM2NP Marseille) and D ominique de Ligny (IGC E rlangen) for stimulating discussions on thermodynamics of glasses. Philippe Maugis (IM2NP Marseille) for enlightening discussions on thermodynamics of phase transitions and critical phenomena. Marc G ailhanou (IM2NP Marseille) for invaluable help in numerical computing and Python programming. This work was motivated by discussions in the subgroup "Thermodynamics of Glasses" led by Sophie Schuller (CEA Marcoule), within the French thematic network "Thermodynamique des Matériaux Hautes Températures" (ThermatHT) - GDR CNRS n³584. 


\section{References}

[1] I. Prigogine, R. D efay, Chemical Thermodynamics, Longmans, G reens and Co., 1954.

[2] S. V. Nemilov, Thermodynamic and Kinetic Aspects of the Vitreous State, CRC Press, 1995.

[3] I.S. Gutzow, J.W.P. Schmelzer, The Vitreous State: Thermodynamics, Structure, Rheology, and Crystallization, 2nd ed., Springer Verlag Berlin Heidelberg, 2013.

[4] R.O. D avies, G.O. Jones, Thermodynamic and kinetic properties of glasses, Adv. Phys. 2 (1953) 370-410. doi:10.1080/ 00018735300101252.

[5] P.K. Gupta, C.T. Moynihan, Prigogine-D efay ratio for systems with more than one order parameter, J. Chem. Phys. 65 (1976) 4136-4140. doi:10.1063/ 1.432870.

[6] R.J. Roe, Thermodynamics of the glassy state with multiple order parameters, J. Appl. Phys. 48 (1977) 4085-4091. doi:10.1063/ 1.323434.

[7] J. Kovac, Equilibrium Thermodynamics of the G lass Transition, J. Phys. Chem. 85 (1981) 20602065.

[8] T. V Tropin, J.W.P. Schmelzer, I. G utzow, C. Schick, On the theoretical determination of the Prigogine-D efay ratio in glass transition, J. Chem. Phys. 136 (2012) 124502. doi:10.1063/ 1.3694531.

[9] J. G arden, H. G uillou, J. Richard, L. Wondraczek, Non-equilibrium configurational PrigogineD efay ratio, J. Non-Equilibrium Thermodyn. 37 (2012) 143-177. doi:10.1515/ jnetdy-2011-0036.

[10] J. Möller, I. G utzow, J.W.P. Schmelzer, Freezing-in and production of entropy in vitrification, J. Chem. Phys. 125 (2006). doi:10.1063/ 1.2346673.

[11] J.W.P. Schmelzer, I. Gutzow, The Prigogine-D efay ratio revisited, J. Chem. Phys. 125 (2006) 184511. doi:10.1063/ 1.2374894.

[12] I. Gutzow, J.W.P. Schmelzer, B. Petroff, Phenomenological theories of glass transition: Classical approaches, new solutions and perspectives, J. Non. Cryst. Solids. 354 (2008) 311-324. doi:10.1016/ j.jnoncrysol.2007.07.042.

[13] J.W.P. Schmelzer, I. G utzow, Structural order parameters, the Prigogine-D efay ratio and the behavior of the entropy in vitrification, J. Non. Cryst. Solids. 355 (2009) 653-662. doi:10.1016/ j.jnoncrysol.2008.12.014.

[14] T. V. Tropin, J.W.P. Schmelzer, C. Schick, On the dependence of the properties of glasses on cooling and heating rates: I. Entropy, entropy production, and glass transition temperature, J. Non. Cryst. Solids. 357 (2011) 1291-1302. doi:10.1016/ j.jnoncrysol.2010.11.111.

[15] T. V. Tropin, J.W.P. Schmelzer, C. Schick, On the dependence of the properties of glasses on cooling and heating rates II: Prigogine-D efay ratio, fictive temperature and fictive pressure, J. Non. Cryst. Solids. 357 (2011) 1303-1309. doi:10.1016/ j.jnoncrysol.2010.12.005.

[16] J.L. Garden, H. Guillou, J. Richard, L. Wondraczek, Affinity and its derivatives in the glass transition process, J. Chem. Phys. 137 (2012) 4-6. doi:10.1063/ 1.4733333.

[17] C. Cunat, The D NLR Approach and Relaxation Phenomena. Part I - Historical Account and 
D NLR Formalism, Mech. Time-D ependent Mater. 5 (2001) 39-65.

[18] A. A haroune, P. Marceron-Balland, C. Cunat, The D NLR approach and relaxation phenomena. Part II - Application: Volume recovery of PVAc, Mech. Time-Dependent Mater. 5 (2001) 345377. doi:10.1023/ A:1012520720199.

[19] A. Lion, J. Peters, S. Kolmeder, Simulation of temperature history-dependent phenomena of glass-forming materials based on thermodynamics with internal state variables, Thermochim. Acta. 522 (2011) 182-193. doi:10.1016/ j.tca.2010.12.017.

[20] P. Maugis, F. D anoix, H. Zapolsky, S. Cazottes, M. Gouné, Temperature hysteresis of the orderdisorder transition in carbon-supersaturated $\alpha$-Fe, Phys. Rev. B. 96 (2017) 214104. doi:10.1103/ PhysRevB.96.214104.

[21] P. Maugis, Ferrite, martensite and supercritical iron: A coherent elastochemical theory of stressinduced carbon ordering in steel, Acta Mater. 158 (2018) 454-465.

doi:10.1016/ j.actamat.2018.08.001.

[22] H. Eyring, Viscosity, Plasticity, and Diffusion as Examples of Absolute Reaction Rates, J. Chem. Phys. 4 (1936) 283-291. doi:10.1063/ 1.1749836.

[23] J. Frenkel, Kinetic Theory of Liquids, Oxford University Press, Oxford, 1946.

[24] N. Hirai, H. Eyring, Bulk viscosity of polymeric systems, J. Polym. Sci. 37 (1959) 51- 70. doi:10.1002/ pol.1959.1203713104.

[25] B. Wunderlich, D.M. Bodily, M.H. Kaplan, Theory and measurements of the glasstransformation interval of polystyrene, J. Appl. Phys. 35 (1964) 95-102. doi:10.1063/ 1.1713105.

[26] R.P. Smith, Equation of state and the thermodynamic properties of liquid polymers: Application of the Hirai-Eyring model, J. Polym. Sci. Part A-2 Polym. Phys. 8 (1970) 1337-1360. doi:10.1002/ pol.1970.160080807.

[27] T. Somcynsky, R. Simha, Hole Theory of Liquids and Glass Transition, J. Appl. Phys. 42 (1971) 4545-4548. doi:10.1063/ 1.1659821.

[28] G.P. Johari, Configurational and residual entropies of nonergodic crystals and the entropy's behavior on glass formation, J. Chem. Phys. 132 (2010) 124509. doi:10.1063/ 1.3364999.

[29] M. Hillert, Phase equilibria, phase diagrams and phase transformations: their thermodynamic basis, 2nd ed., Cambridge University Press, 2007.

[30] Y. Kraftmakher, Equilibrium vacancies and thermophysical properties of metals, Phys. Rep. 299 (1998) 79-188. doi:10.1016/ S0370-1573(97)00082-3.

[31] H.L. Lukas, S.G. Fries, B. Sundman, Computational Thermodynamics: The Calphad Method, Cambridge University Press, 2007. doi:10.1017/ CBO9780511804137.

[32] P.W. Guan, Z.K. Liu, A physical model of thermal vacancies within the CALPHAD approach, Scr. Mater. 133 (2017) 5-8. doi:10.1016/ j.scriptamat.2017.02.002.

[33] C.H.P. Lupis, Chemical Thermodynamics of Materials, P T R Prentice-Hall, Inc., Englewood Cliffs, New-Jersey, 1983. 
[34] H.E. Stanley, Introduction to Phase Transitions and Critical Phenomena, Oxford University Press, 1971.

[35] M. Hillert, How To Select Axes for a Phase Diagram, J. Phase E quilibria. 18 (1997) 249- 263.

[36] A. Milchev, I. G utzow, Thermodynamic Behaviour of a Simple Liquid- MFA with non-additive Lateral Interactions, Phys. Chem. Liq. 11 (1981) 25-46. doi:10.1080/ 00319108108079094.

[37] Y. Kraftmakher, Lecture Notes on Equilibrium Point D efects and Thermophysical Properties of Metals, World Scientific, 2000.

[38] L.D. Lucas, Liquid D ensity Measurements, in: R.A. Rapp (Ed.), Tech. Met. Res. IV, Interscience Publishers, New York, 1970.

[39] S.S. Chang, A.B. Bestul, Heat capacity and thermodynamic properties of o-terphenyl crystal, glass, and liquid, J. Chem. Phys. 56 (1972) 503-516. doi:10.1063/ 1.1676895.

[40] H. Leyser, A. Schulte, W. D oster, W. Petry, High-pressure specific-heat spectroscopy at the glass transition in o -terphenyl, Phys. Rev. E. 51 (1995) 5899-5904. doi:10.1103/ PhysRevE.51.5899.

[41] A. Tölle, Neutron scattering studies of the model glass former ortho -terphenyl, Reports Prog. Phys. 64 (2001) 1473-1532. doi:10.1088/ 0034-4885/ 64/ 11/ 203.

[42] M. Naoki, S. Koeda, Pressure-volume-temperature relations of liquid, crystal, and glass of oterphenyl: excess amorphous entropies, and factors determining molecular mobility, J. Phys. Chem. 93 (1989) 948-955. doi:10.1021/ j100339a078.

[43] J.N. Andrews, A.R. Ubbelohde, Melting and crystal structure: the melting parameters of some polyphenyls, Proc. R. Soc. London. Ser. A. Math. Phys. Sci. 228 (1955) 435- 447. doi:10.1098/ rspa.1955.0059.

[44] R.J. G reet, D. Turnbull, G lass Transition in o $\square$ Terphenyl, J. Chem. Phys. 46 (1967) 1243-1251. doi:10.1063/ 1.1840842.

[45] J. Bartoš, J. Krištiak, A phenomenological model of volumetric and free volume hole properties in supercooled liquids: the ortho-terphenyl case, J. Phys. Chem. B. 104 (2000) 5666-5673. doi:10.1021/ jp993703f.

[46] B.D. Malhotra, R.A. Pethrick, Positron annihilation studies of supercooled liquids. orthoterphenyl, J. Chem. Soc. Faraday Trans. 2.78 (1982) 297. doi:10.1039/ f29827800297.

[47] S.P. Verevkin, Thermochemistry of substituted benzenes. Experimental standard molar enthalpies of formation of 0-, m-, and p-terphenyls and 1,3,5-triphenylbenzene, J. Chem. Thermodyn. 29 (1997) 1495-1501. doi:10.1006/ jcht.1997.0265.

[48] C. Tsonopoulos, D. Ambrose, Vapor-Liquid Critical Properties of Elements and Compounds. 3. Aromatic Hydrocarbons, J. Chem. Eng. D ata. 40 (1995) 547-558. doi:10.1021/ je00019a002.

[49] H. Tanaka, General view of a liquid-liquid phase transition, Phys. Rev. E. 62 (2000) 6968-6976. doi:10.1103/ PhysRevE.62.6968. 